\title{
Irreducible Traumatic Anterior Shoulder Dislocation Secondary to Both Subscapularis and Long Head of Biceps Tendon Interposition-A Case Report
}

\author{
Zachariah E. Miles, Robert J. Gordon, Matthew P. R. Wilkinson
}

Department of Orthopaedics, The Townsville Hospital, Townsville, Australia.

Email: zacmiles@hotmail.com

Received March 15 ${ }^{\text {th }}$, 2012; revised April 20 ${ }^{\text {th }}$, 2012; accepted May $18^{\text {th }}, 2012$

\begin{abstract}
An Irreducible dislocation of the shoulder is an uncommon event. When it does occur, blocks to reduction can include bone, labrum, rotator cuff musculature or tendon. Patients older than 40 at the time of initial dislocation are at increased risk of sustaining a concomitant rotator cuff tear. We present a case of an irreducible anterior shoulder dislocation due to interposition of both subscapularis tendon and a posteriorly dislocated long head of biceps. Both Computed Tomography (CT) and magnetic resonance imaging (MRI), along with intraoperative findings are discussed. Conclusion: We would advocate maintaining a low threshold for MR imaging post shoulder dislocation in the older population, when there is radiological or clinical concern regarding the integrity of the rotator cuff, and also to evaluate whether a concentric reduction of the shoulder joint has been achieved.
\end{abstract}

Keywords: Irreducible Shoulder Dislocation; Long Head of Biceps; Subscapularis

\section{Introduction}

Dislocation of the shoulder joint is a common presenting complaint to Emergency Departments. In greater than $95 \%$ of cases, the dislocation is in an anterior direction [1]. Patients older than 40 at time of initial dislocation are at increased risk of associated rotator cuff tears [2,3]. Dislocation of the long head of biceps tendon during glenohumeral dislocation also occurs infrequently, and when it does, it is most often in a medial direction [4]. Impediments to successful reduction are many, and can include bone, labrum, rotator cuff, or long head of biceps tendon [5]. A recent article also describes interposition of the musculocutaneous nerve as the cause of an irreducible dislocation [6]. We present a case of, and discussion around, an irreducible glenohumeral dislocation seconddary to interposition of both subscapularis tendon and a posteriorly dislocated long head of biceps tendon. This was confirmed on both CT and MRI, together with operative findings, which demonstrated a massive acute rotator cuff tear. Few cases of either subscapularis interposition, or posterior dislocation of the long head of biceps tendon have been described. To date, in the literature there are no reported cases of these combined blocks to successful reduction of an anterior glenohumeral joint dislocation. Awareness of the potential for such associated injuries is important in cases of persistent subluxa- tion or persisting pain and functional deficit.

\section{Case}

A 64 year old male presents to the emergency department with an isolated injury to his right shoulder, which he sustained the day prior, after having a fall clearing debris post cyclone Yasi. He clinically had an anterior dislocation of his shoulder, which was reduced in the emergency department-see post reduction films, (Figure 1). It is clear in the post reduction films that the head of the humerus is positioned superiorly, with minimal subacromial space.

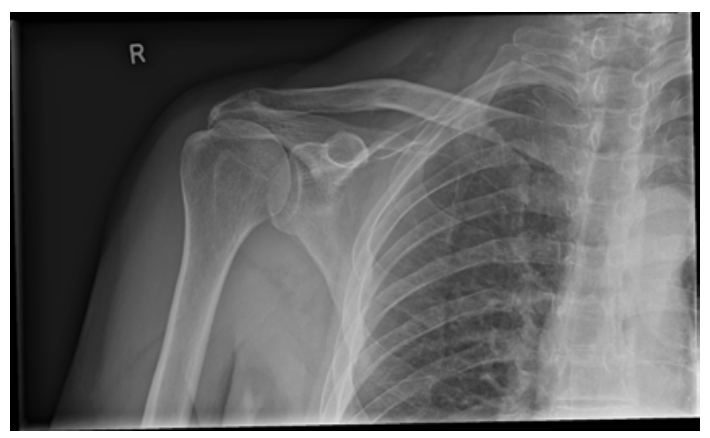

Figure 1. Initial post-reduction films showing apparent glenohumeral reduction but significant loss of acromiohumeral space. 
He was seen in fracture clinic one week post injury. He was neurovascularly intact, however still had signifycant pain and his range was limited to 20 degrees of abduction and flexion. He was referred to physiotherapy and a review appointment was booked. At his review 3 weeks later, he showed no improvement. Initial X-rays were reported as showing a glenoid rim fracture and as such CT scan was ordered (see Figures 2-3). These demonstrated no injury to the glenoid but a persistent anterior-superior subluxation of the glenohumeral joint. A provisional diagnosis of massive rotator cuff tear was made with associated glenohumeral escape, and an MRI was then ordered as preparation for surgery (Figures 4-6). The MRI confirmed the diagnosis of a massive rotator cuff tear to supraspinatus, infraspinatus and subscapularis, and the long head of biceps tendon had dislocated postero-laterally around the humeral head. Both the long head of biceps tendon as well as the torn subscapularis muscle where interposed within the glenohumeral joint, pushing the humerus anteriorly and preventing full reduction. There was also minimal supraspinatus and infraspinatus atrophy or fatty infiltration (see Figure 6), suggesting the supraspinatus tear was acute as per Thomazeau criteria [7], and Goutallier classification [8].

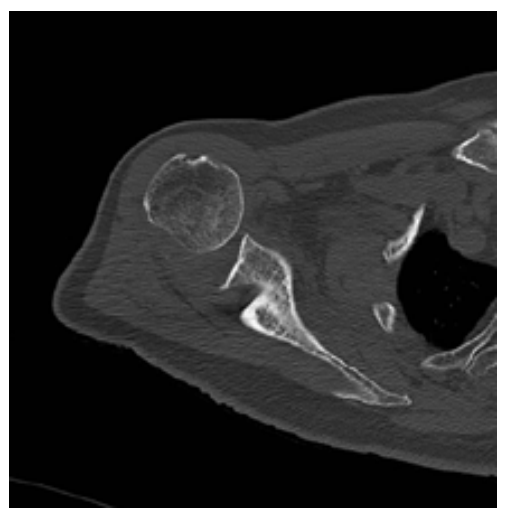

Figure 2. CT scan demonstrating antero-superior subluxation of the humerus.

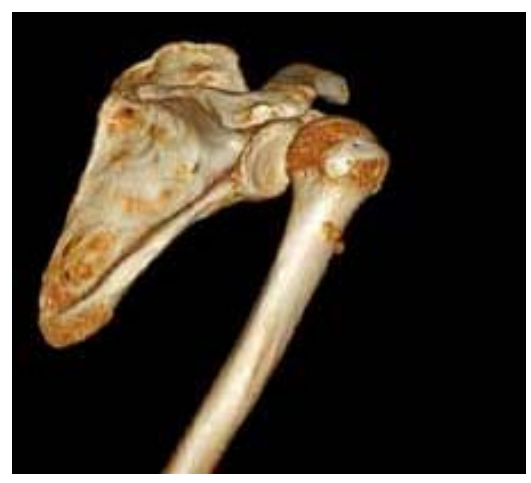

Figure 3. CT scan 3D reconstruction again demonstrating persistent subluxation of the glenohumeral joint.

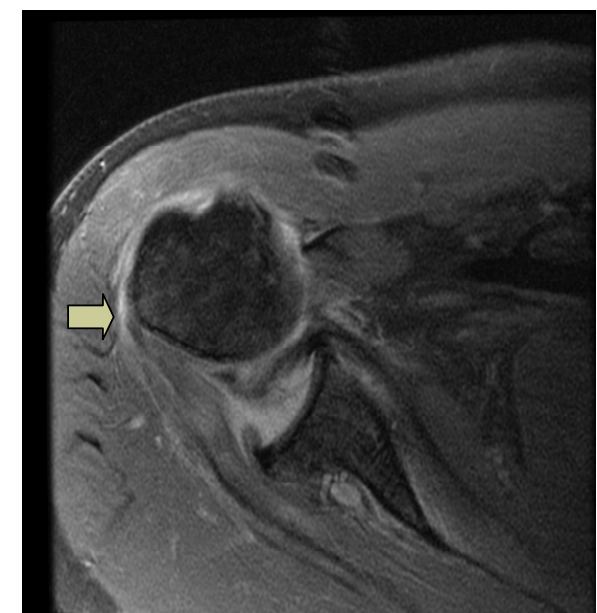

Figure 4. MRI demonstrating subscapularis interposed into the joint with the biceps tendon traversing posteriorly around the humerus (arrow).

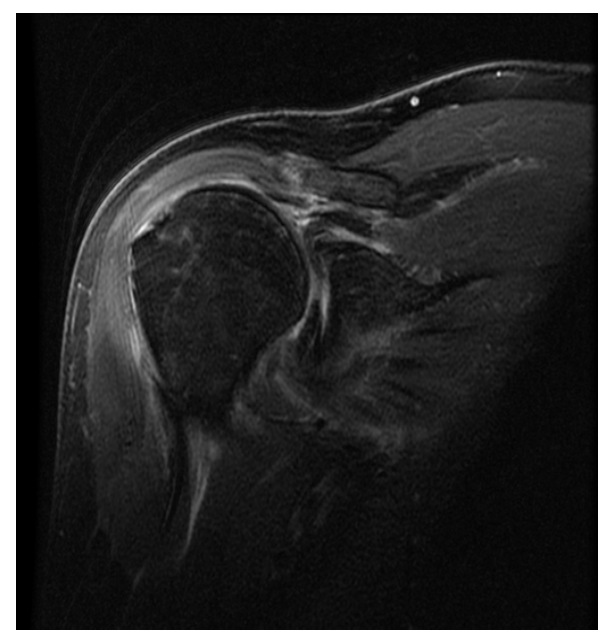

Figure 5. Coronal MRI demonstrating a massive tear to the supraspinatus with the retracted tendon at the rim of the glenoid and subscapularis within the joint.

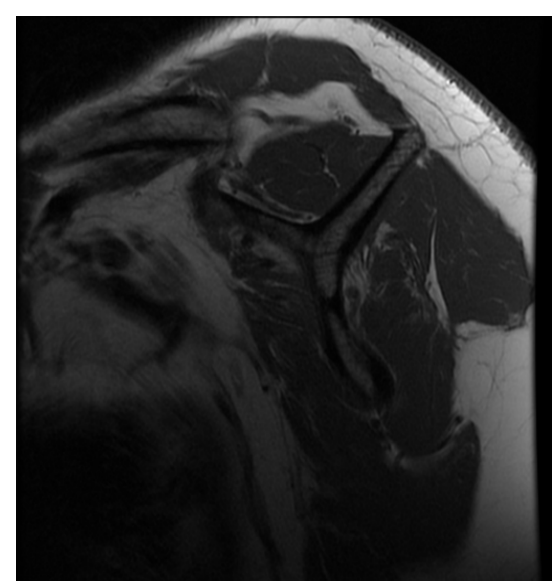

Figure 6. Sagittal MRI demonstrating good muscle bulk to the supraspinatus and infraspinatus. 
At nearly 6 weeks post injury the patient was taken to the operating room and a strap incision was employed to allow surgical access to both the deltopectoral interval as well as a deltoid splitting interval. Incision through the clavipectoral fascia revealed a nude humeral head. The subscapularis was firmly adhered to the glenoid surface, and the long head of biceps had scarred down to the posterior capsule. The subscapularis tendon was removed from the joint and repaired to the lesser tuberosity with multiple anchors. The biceps was tenodesed at the level of the subscapularis repair. The supraspinatus and infraspinatus were mobilised and repaired with a double row modified suture bridge technique (Figures 7-11).

Post-operatively the patient was managed in a sling for 6 weeks. During this time he was allowed to perform pendular exercises as well as wrist and elbow exercises. A post-operative film showed restoration of the acromiohumeral space (Figure 12).

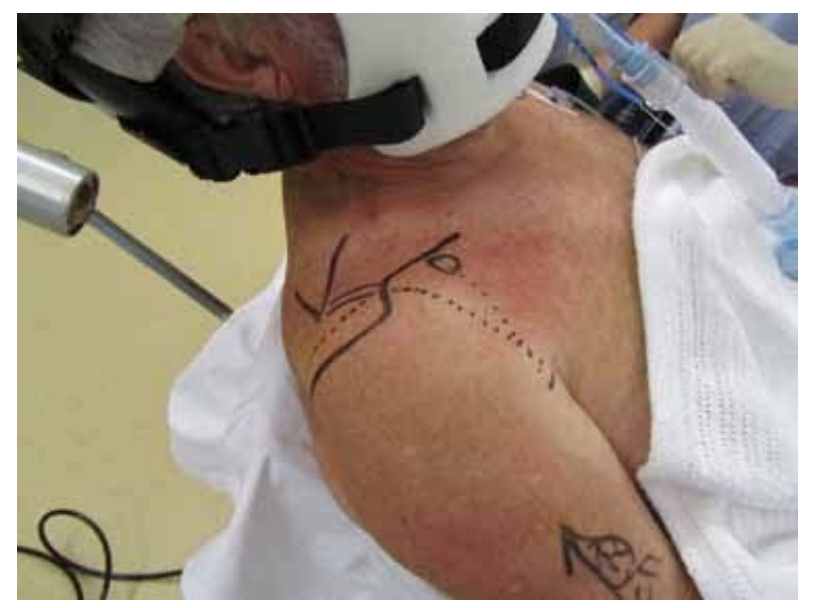

Figure 7. Planned surgical incision marked (deltopectoral groove also marked for reference).

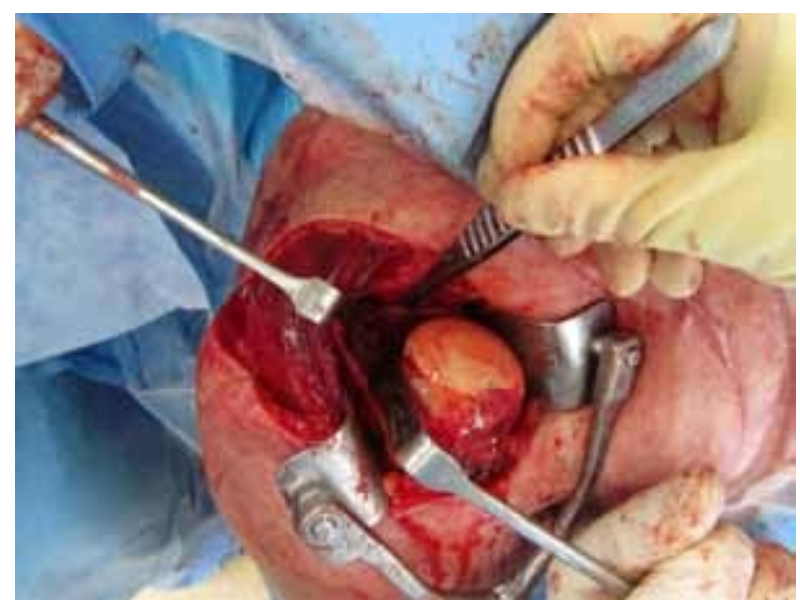

Figure 8. Incision through the deltopectoral interval reveals humeral head devoid of cuff attachments and subscapularis blocking reduction.

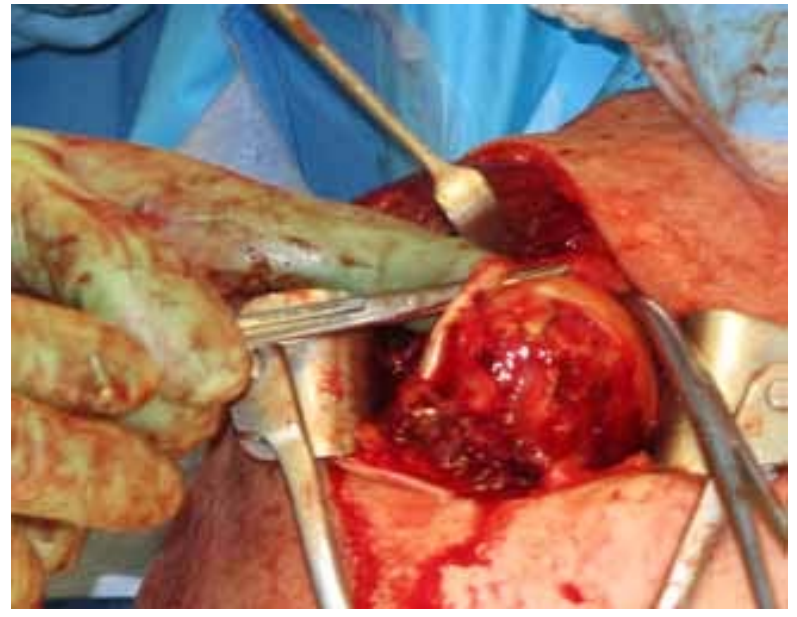

Figure 9. Biceps tendon posteriorly dislocated around humerus.

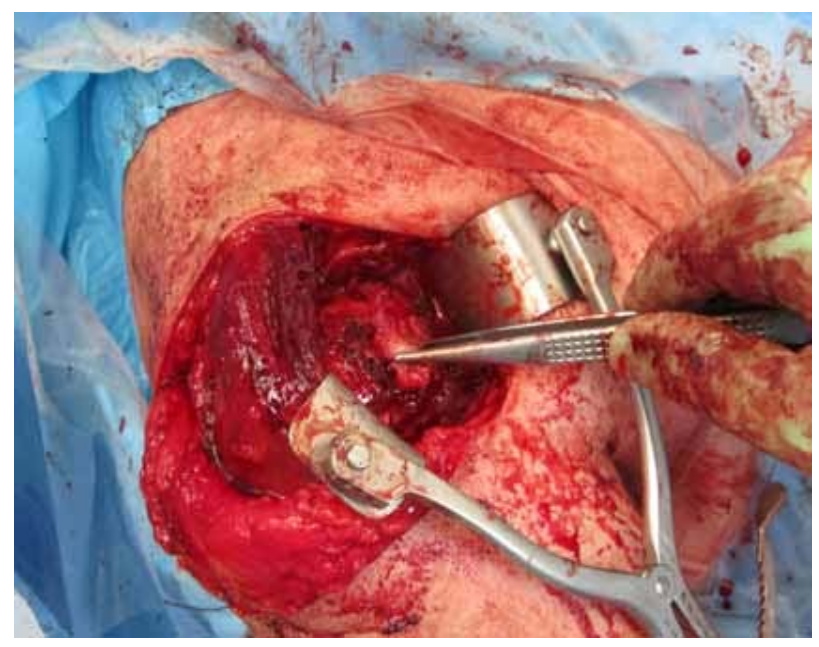

Figure 10. Subscapularis repaired to trough on lesser tuberosity.

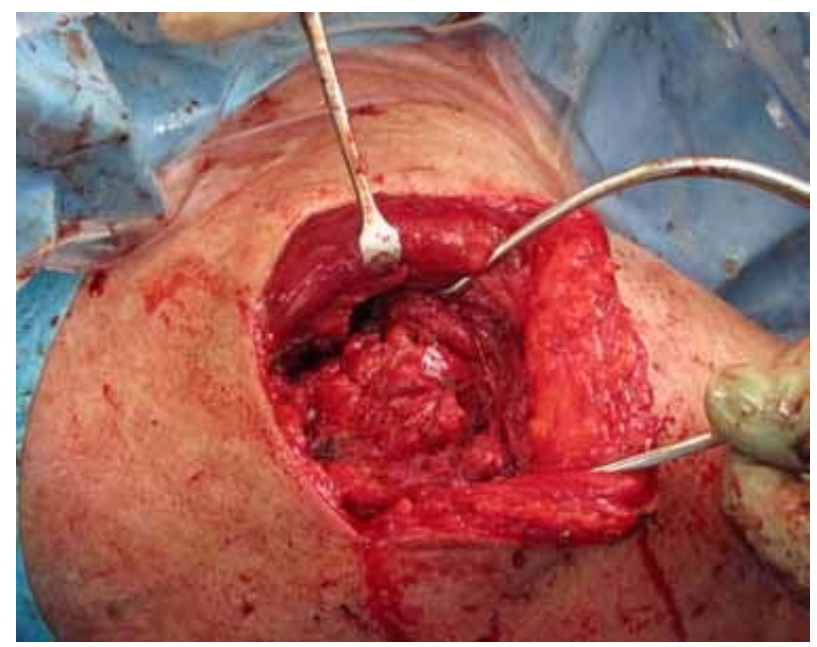

Figure 11. Double row repair of supraspinatus and infraspinatus with suture bridge technique. 


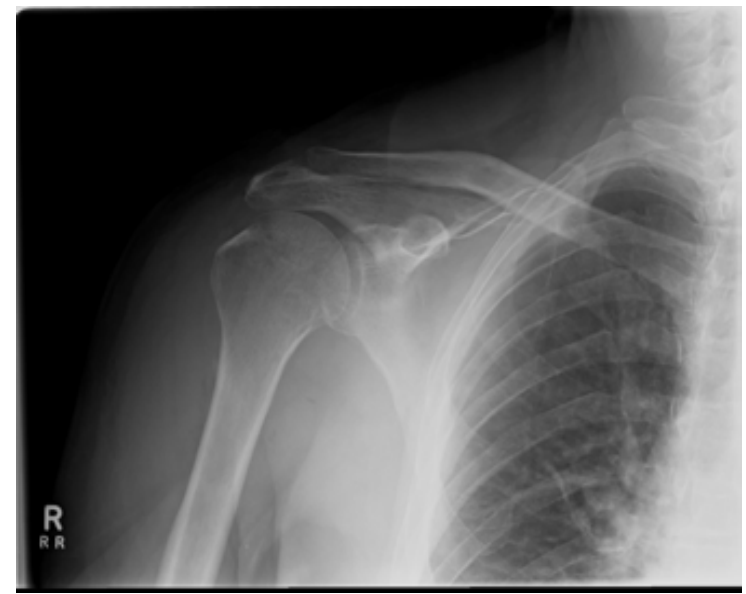

Figure 12. Post-operative $\mathrm{X}$-ray showing restoration of acromiohumeral space.

\section{Discussion}

The shoulder joint is one of the most commonly dislocated joints in the human body. Orthopaedic surgeons are familiar with the consequences of shoulder dislocations in different age groups, and the association with rotator cuff tears in older patients is well recognised [3]. Rarely, however, is thought given to the possibility of entrapped soft tissue after shoulder dislocation. Persistent subluxation of the glenohumeral joint post dislocation can be easily missed by simple radiographs. Although CT identifies associated bony injuries and in our case identified the persistent joint subluxation, it fails to adequately image the associated soft tissue injury and possibly delays definitive management. MRI is a very useful adjunct in evaluating the shoulder post-dislocation in any age group and is particularly useful for imaging the rotator cuff. In this case it identified the associated rotator cuff tears, and the presence of the subscapularis interposed in the joint with the postero-laterally dislocated long head of biceps. It was also valuable in assessing the quality of the supraspinatus and infraspinatus muscle belly and the possibility of a successful repair.

Subscapularis tendon interposition post dislocation has only rarely been reported in the literature [11,12]. When the subscapularis tendon is ruptured the biceps tendon can dislocate medially and also interpose in the joint. For a postero-lateral dislocation of the long head of biceps tendon to occur, lateral stabilising structures of the rotator cuff need to be deficient also [8,9]. This lack of lateral restraint then allows the biceps tendon to move around the lateral margin of the humerus, and into the posterior aspect of the glenohumeral joint. In our case, a shoulder dislocation combined with a massive rotator cuff tear allowed the tendon to dislocate. Other studies have shown posterior dislocation of the long head of biceps tendon into the shoulder joint $[4,5,9,10]$, but due to the rarity of this condition, the literature is limited to sporadic case reports.

To the best of our knowledge, we believe this to be the first case reported to demonstrate the rare finding of a postero-lateral dislocation of the long head of the biceps in association with the subscapularis both contributing to mechanical soft tissue block to reduction of a dislocated shoulder.

Strobel et al. (2002) make the pertinent comment, "on MR imaging in patients after shoulder dislocation, the position and course of the long head of biceps tendon should be evaluated so that a posterior displacement... will not be overlooked" [10]. A posteriorly dislocated biceps tendon can be missed even during open surgery leading to a poor outcome. We would advocate maintaining a low threshold for MR imaging post shoulder dislocation in the older population, when there is radiological or clinical concern regarding the integrity of the rotator cuff, and also to evaluate whether a concentric reduction of the shoulder joint has been achieved.

\section{REFERENCES}

[1] S. Cutts, M. Prempeh and S. Drew, "Anterior Shoulder Dislocation," Annals of the Royal College of Surgeons of England, Vol. 91, No. 1, 2009, pp. 2-7. doi:10.1308/003588409X359123

[2] L. Stayner, et al., "Shoulder Dislocations in Patients Older than 40 Years of Age," Orthopedic Clinics of North American, Vol. 31, No. 2, 2000, pp. 231-239. doi:10.1016/S0030-5898(05)70143-7

[3] R. Neviaser, T. Neviaser and J. Neviaser, "Concurrent Rupture of the Rotator Cuff and Anterior Dislocation of the Shoulder in the Older Patient," Journal of Bone and Joint Surgery, Vol. 90, No. 9, 1998, pp. 1308-1311.

[4] V. Cervilla, et al., "Medial Dislocation of the Biceps Brachii Tendon: Appearance at MR Imaging,” Radiology, Vol. 180, No. 2, 1991, pp. 523-526.

[5] M.S. Day, et al., "Irreducible Anterior and Posterior Dislocation of the Shoulder Due to Incarceration of the Biceps Tendon,” International Journal of Shoulder Surgery, Vol. 4, No. 3, 2010, pp. 83-85. doi:10.4103/0973-6042.76970

[6] R. Gudena, et al., "Irreducible Shoulder Dislocation: A Word of Caution," Orthopaedics and Traumatology: Surgery and Research, Vol. 97, No. 4, 2011, pp. 451-453. doi:10.1016/j.otsr.2011.02.004

[7] H. Thomazeau, et al., "Atrophy of the Supraspinatus Belly,” Acta Orthopaedica Scandinavica, Vol. 67, No. 3, 1996, pp. 264-268. doi:10.3109/17453679608994685

[8] D. Goutallier, et al., "Fatty Muscle Degeneration in Cuff Repairs. Pre- and Post-Operative Evaluation by CT Scan," Clinical Orthopaedics and Related Research, Vol. 304, 1994, pp. 78-83.

[9] P. J. Mullaney, et al., "Posterior Dislocation of the Long Head of Biceps Tendon: Case Report and Review of the Literature,” Skeletal Radiology, Vol. 36, No. 8, 2007, pp. 
779-783. doi:10.1007/s00256-007-0285-7

[10] K. Strobel, T. C. Treumann and B. Allgayer, "Posterior Entrapment of the Long Biceps Tendon after Traumatic Shoulder Dislocation: Finding on MR imaging," American Journal of Roentgenology, Vol. 178, No. 1, 2002, pp. 238-239.

[11] S. Connolly, et al., "Irreducible Anterior Dislocation of the Shoulder Due to Soft Tissue Interposition of Subscapularis Tendon,” Skeletal Radiology, Vol. 37, No. 1, 2008, pp. 63-65. doi:10.1007/s00256-007-0370-y

[12] S. H. Bridle and B. D. Ferris, "Irreducible Acute Anterior Dislocation of the Shoulder: Interposed Subscapularis," Journal of Bone \& Joint Surgery, British Volume, Vol. 72, 1990, pp. 1078-1079. 